TWO CASES OF EXTENSIVE

\title{
ARTERIAL OBSTRUCTION
}

FroN

SEPARA'TED CARDIAC VEGE'TA'TIONS,

FOLLOWED BY

GANGRENE OF THE LOWER EXTREMITIES AND DEA'TH.

15Y

S. J. GOODFELLOW, M.D. IOND., F.R.C.P., PHYSICIAN TO AND LECTURER ON MEDICINE AT THE MIDDLESEX HOSPITAL.

Received April 23rd.-Read June 24th, 1862.

IN presenting the two following cases to the Society, it is not my intention to enter into the subject of arterial obstruction generally. I am quite aware that instances of plugging of arteries by so-called vegetations from the heart are not uncommon. The extent, however, to which the plugging took place, the number of vessels involved, the morbid changes in and around the walls of the vessels at the seat of obstruction, and the consequences which ensued, appear to me to give a peculiar interest to these cases, and to render them worthy of being placed on record.

Case 1.-Maria Chitton, a sempstress, æt. 30 years, was admitted into the Middlesex Hospital under my care, January 29th, 1861. She was well-formed, of middle-height, and moderately stout and muscular. She was unmarried, but had cohabited with a man for thirteen years up to about six months before her admission, when he left her. This 
desertion affected her much, and she abandoned herself to a very irregular and dissolute life. She never had syphilis. Twelve years ago, when at the age of eighteen years, she had a severe attack of rheumatic fever, after which she suffered more or less from palpitation and dyspnœa ; but with this exception and occasional "colds," her health was tolerably good up to about six months ago. Since that she has suffered more than before from palpitation and dyspnœa, being obliged at times to remain in bed for several days consecutively from apparently fresh attacks of rheumatism.

About a week before her admission she was suddenly seized with severe pain in the calf of the left leg and in the ankle and a sensation of numbness in the toes. There was some swelling around the ankle, but no redness; there was neither swelling nor redness in the calf of the leg. The lightest touch in the latter situation gave rise to intense pain.

On admission there was still intense pain in the left ankle and calf of the leg, but, as before admission, there was no redness, and the limb was of the normal temperature. The numbness of the toes was still present. The countenance was anxious, and expressive of great suffering. There was no pain in any other situation, not even along the course of the principal vessels of the limb under pressure. Pulsation could be felt in the femoral artery, and also in the course of the popliteal, but only indistinctly. There was a brownish discoloration of the skin of the lower and inner part of both thighs and inner side of the knees, and upon these discoloured parts there were minute cuticular scales. The eruption resembled that of pityriasis. There were no nodes discoverable. The pulse in the right wrist was moderately full, but soft, while that in the left was extremely small ; number of pulsations, 90 . The heart's impulse was unusually strong and heaving. Extent of cardiac dulness was considerably increased, both vertically and transversely. Systolic sound short, and accompanied by a harsh murmur, which was heard loudest near the apex, and upwards and outwards under the nipple; the second 
sound was absent. The respiration was hurried; there was no cough; percussion-resonance dull over the base of the right lung, where the inspiratory murmur was harsh and blowing, and the respiration was accompanied by a medium sub-crepitant rhonchus. Some dry crumpling, as from emphysema, at anterior edge of upper lobe, and at apex. Resonance normal in other parts of this side; it was also normal over the whole of the left side, but the respiration at the base posteriorly of the left lung was accompanied also by a medium sub-crepitant rattle. The urine was in normal quantity, had a specific gravity of 1015, was acid, and gave no precipitate by heat and $\mathrm{NO}_{5}$.

February 2nd.-Notwithstanding the administration of opiates in large doses internally, and their topical application, she has had scarcely any sleep, except for a few minutes at a time, since her admission, on account of the pain in the calf of her leg, which has sometimes been so agonising as to make her cry aloud. On the 31st of January (second day after admission) a purplish, irregular patch was noticed on the dorsum of the left foot; it occupied a space of about two inches in length by from an inch to an inch and a half in breadth; it did not disappear on pressure, and was not raised above the level of the surrounding skin. The temperature of the left foot was less than that of the right. This patch has been slowly and gradually extending in all directions. The left foot is now considerably colder than the opposite one. Pulse 120, extremely small, and weak.

6th.-Dry gangrene on the foot has considerably extended; it now occupies nearly the whole of the upper part of the foot. To-day a large bulla was observed on the inner side of the leg, a little above the ankle; it was filled with a dark-green fluid. This foot was several degrees colder than the right, and its sensibility almost lost.

7th.-The left foot is now odematous. The bleb has burst, and the surrounding skin is of a greenish colour. The femoral artery can be felt pulsating feebly. Breathing is short and jerking; number of respirations, 41 in the minute ; constant orthopnœa.

vol. XLV. 
11th.-Between this and the last report the dry gangrene has been slowly extending, and involves now nearly the whole of the foot; there is as yet no evidence of any separation of the gangrenous part. The moist gangrene above has also extended, numerous blebs having formed at the periphery of the first patch, and a succession of them invading from day to day the neighbouring sound skin; the pain in the calf of the leg is much less, but there is now great tenderness to the touch or on motion, with preternatural heat in the popliteal space, and in the evening she complained of much pain and numbness in the right arm; there is no discoloration; the radial pulse at this side is scarcely perceptible.

13th.-The pain in the right arm was so great as completely to deprive her of sleep; prostration is now considerable; expression of countenance is extremely anxious and careworn; the temperature of this arm is somewhat lower than that of the left; no pulsation can now be felt at the wrist or in the course of the brachial artery; only a very slight pulsation can be felt over the axillary artery. From this time she began to sink rapidly, and died at $2 \cdot 30$ p.m. For nearly an hour previously to her death she was insensible, but there were no convulsions. Occasionally during her stay in the hospital she was delirious, but the delirium was never of a violent character.

Autopsy (thirty-five hours after death; weather cold).Rigidity marked, but less so in the left lower extremity; body well nourished, upwards of an inch of sub-cutaneous fat over the abdomen; the left foot and four or five inches of the left leg were in a state of dry gangrene.

Head.-About an ounce and a half of clear fluid at the base of the brain. A superficial sub-arachnoid ecchymosis, of about the size of a sixpence, on the upper part of the right hemisphere. The brain and its membranes in other respects healthy.

Chest.-Both lungs were non-adherent. Weight of right, $20 \mathrm{oz}$; that of left, $21_{4}^{3} \mathrm{oz}$; they were crepitant throughout, and very œdematous posteriorly, but there 
was nowhere any consolidation; considerable emphysema along the anterior margins.

Heart much enlarged; the right cavities much enlarged, and both filled with dark coagula and grumous blood. The aortic valves were healthy; some atheroma around the orifices of the coronary arteries, but the aorta above was free from disease ; left ventricle at the base measured $4 \frac{3}{4}$ in., the thickness of its wall, 1 in. The curtains and edges of the mitral valve were covered with large vegetations $(a)$, which projected more than an inch beyond the edges of the valve, and extended a considerable distance along the lining membrane of the auricle, and almost filled the appendix $(b)$; the one by which this mass terminated was as large as a small hazel-nut; the superficial vegetations were so soft as scarcely to bear manipulation, while those at the base were made up of firmer material. The substance of the organ beneath and around the seat of the vegetations was infiltrated with a yellowish, puriform matter, which in some parts was exceedingly soft. In other parts $(c)$ of the wall of the ventricle similar deposits were observed, and these also were friable. The carneæ columnæ were much enlarged, and the cordæ tendineæ thickened.

Arterial system.-In the right brachial artery $(d)$ there was a firm coagulum, which extended from the point at which the anastomotica magna is given off to the bend of the arm, where the artery was separated. How far it extended beyond was not ascertained. The right and left carotids were free from coagula, as were also the arteries of the brain, so far as they were examined. Arch and thoracic portion of aorta free from disease. The abdominal aorta at the point of origin of the coliac axis $(e)$ was filled with a firm coagulum, as was also the cœliac axis itself, $(f)$, but no vegetation could be discovered in these coagula. The coagulum in this portion of the aorta extended a little below the origin of the renal arteries. At the bifurcation of the aorta there were several yellowish-white masses, apparently of the same structure as the vegetations observed in the heart, which were partially surrounded by blood- 
coagula, and this mixed mass extended for about half an inch into the common iliac on each side $(h, h)$. Below this these vessels were filled by a blood-coagulum as far as their division, where there were other deposits of the same character as those seen in the mitral valve, surrounded by blood-coagulum, especially in the internal iliacs on both sides. At the points above mentioned $(f, g, h, i)$ the adhesion between the vessels and the contained deposits and coagula was considerable, and the walls of the arteries were much thickened, and surrounded by and imbedded in dense fibrous tissue, so that the vessels could be removed from their situation only by careful dissection. Surrounding the deposits in the right internal iliac artery, the coats of the vessel were not only thickened, but were separated by a curdy, puriform matter $(j)$, and in one part there was a cavity of the size of a horse-bean, filled with this matter; in fact, a small abscess. The same changes were observed in the left internal iliac, but the cavity was smaller. The external iliacs and femoral on both sides, as far as the origin of the profunda, were filled with coagula; at the origin of this latter vessel $(k)$ the inner coat was separated from the outer coats by the same curdy, puriform matter as was observed between the coats of the internal iliacs; below this point, on the right side, the profunda was pervious. On the left side, both in the femoral and profunda, this separation of the coats, was extensive and penetrated for a considerable distance along the femoral; and about two inches below the origin of the profunda there was another fibrinous vegetation observed, and here likewise the inner layers were separated from the outer layers by a thick, puriform, curdylooking matter ; from this point the vessel was solid down to the division of the popliteal, where there was another vegetation observed, and where the walls were found in the same state. The large veins of the legs were filled with a firm coagulum, which adhered firmly to these vessels. (See Plate IX.)

Abdomen.-Liver, 70 oz. The left lobe adherent by recent lymph, and the capsule considerably thickened. 
The inflammation was greatest where the left lobe came in contact with the diseased spleen. The rest of the organ appeared healthy, and the capsule of the right lobe was not thickened.

Spleen $(l)$ measured seven inches long by three wide and two thick; its outer surface was connected to its surrounding parts by recent adhesions; the whole organ was much softened; at its lower extremity was a buff-coloured deposit; the structure of the organ in this situation was exceedingly friable, and seemed to be converted into a puriform mass. At the upper extremity there was another deposit, about half an inch thick and one inch in width; in the centre of the organ there were also numerous deposits of the same character.

Kidneys.-There were numerous cuneiform deposits in both these organs. Most of them were of a pale-yellow colour and of firm consistence, their bases, at the outer surface of the kidney were depressed, and the largest measured about half an inch in diameter; right kidney weighed $6 \frac{1}{4} \mathrm{oz}$., and contained five or six deposits. The left kidney, being attached to the preparation, was not weighed, but seemed to be of the same size as the right.

CASE 2.-Margaret Holloway, æt. 17, a servant of all work, admitted under the care of Dr. Stewart, March 5th, 1861.

This girl was of a spare habit of body, and her muscles were small and flabby. She was born, and had always resided, in London. She had smallpox when an infant, but no other disease, so far as she knows, until the age of fourteen (three years before her admission), when she had rheumatic fever. This attack is reported to have been complicated with pneumonia, but not with any cardiac affection. She had another attack of acute rheumatism a year and a half after the first. In this attack the heart was affected, and ever since she suffered more or less from dyspnœa on any unusual exertion, and severe pain in the chest. On the evening of the 1st of March (five days 
before admission) she was seized somewhat suddenly with severe pain in the left side, which compelled her to leave her employment. On the following day, in addition to the severe pain in the side, she suffered from headache and a feeling of great depression. These symptoms continued up to the time of her admission into the hospital. She had always lived well, but had been much exposed to cold, and had frequently worked in damp clothes, to which she ascribed her rheumatic attacks. She never menstruated. There were no marks of scrofula.

On her admission the face was pale, features were pinched, and the expression anxious. The skin was not preternaturally warm; the pulse 96 , slightly rebounding, and small ; tongue somewhat pale, and slightly coated at the root; some appetite; bowels represented to be duly moved every day. Cardiac dulness from the body of the second rib to the upper border of the fifth rib ; total of vertical dulness, four inches and three quarters; a rough murmur, seemingly diastolic, immediately following sharp second sound; it was heard from the second sterno-costal articulation downwards to the base of the fourth rib, and from the cartilage of this rib outwards to infra-axillary region; there was also a loud systolic murmur which entirely masked the first sound, and continued even during a portion of the second sound ; slight fremitus between the third and fourth ribs. Percussion under the right clavicle slightly deficient in resonance, and the expiration was prolonged and blowing; increased vocal resonance as far as the fourth rib. On the left side the percussionresonance, and the respiratory murmur, varied little from those of health. There was no morbid symptom referable to the abdomen or to the brain. She had no pain in any of the joints or in the limbs. The urine was somewhat scanty and high coloured. These symptoms progressively abated, and she appeared better in all respects until mid-day on the 8th, when she had a severe rigor of about an hour's duration, which was followed by a fainting fit, after that by free perspiration and also pain in the left knee-joint, and pain and swelling in the inner aspect of the right knee. Pulse 102, 
moderately full, but jerking and easily compressed; respirations about 32, variable in frequency, deep, heaving, thoracic. The murmur at the base of the heart much less loud; that at the fourth rib was unchanged. Bowels open.

March 22nd.-From the 8th to the 20th the improvement was considerable, but on the morning of that day she awoke with a feeling of feverishness; the skin was dry and pungently hot; the tongue was dry and brown. On the previous evening she complained of pain in the right foot and toes, and on this morning (the 22nd) she had pain in the right hand, which was increased by pressure; pulse was variable, from the rate of 96 to that of 181, still moderately full, but jerking and very compressible. Respiration also very variable, from the rate of 14 to 28 in a minute, and entirely thoracic. The heart's sounds as before.

25th.-On the 23rd and yesterday she felt much better, but to-day complains much of pain in the knees, especially the left, which is puffy and tender, and its superficial vessels are enlarged; is perspiring freely; tongue clammy, and coated with a yellow fur at the root.

April 23rd (seven weeks since her admission).-Since last report she suffered from occasional pain in the knees and ankles, which never lasted long, and was seldom accompanied by swelling, and never by redness; but she was never entirely free from uneasiness in these joints, and without any manifest cause she had not progressed favorably, and continued to become weaker, more pallid, and more emaciated, in spite of tonics and a generous diet.

On the evening, however, of this day she was seized suddenly with pains of the most intense character in the lower extremities, occasionally lessening in severity, but recurring with former violence. The pain was principally below the knees. There was inability to flex the leg or move it in any direction; sensibility was gone below the knees and for about six inches above them, higher on the anterior than on the posterior aspect. No spasmodic muscular action could be seen or felt, nor were there any convulsive twitchings. The temperature was palpably much 
below that of the surface of the body; quite cold at the feet, the warmth gradually increasing on approaching the trunk. The manner was excited, and she made frantic gestures and exclamations in her suffering. The skin was intensely hot, face flushed, breathing hurried, and heart's action regular, rapid, and vehement. The abnormal cardiac murmurs, before existing, were much intensified. There was no pain in the head, no perrersion of the special senses, pupils were of normal size and readily influenced by changes of lights, and there was no tenderness along the spine.

25th.- She continued much in the state above described, with occasional remissions of the pain from the internal administration and local application of opiates. There is now complete loss of sensation from the middle of the left thigh downwards, and also from the corresponding part of the right thigh, except at the knee, which is painful under pressure. Temperature below the knee markedly deficient. No pulsation in the popliteal or femoral arteries; no œdema of the limbs, but the superficial veins on the dorsum of each foot are enlarged and prominent. On the plantar aspect, and at the extremity of the great and second toes, there are patches of blue discoloration. Feet are dry and harsh ; pulse 108, full, and very firm; heart's action turbulent. The abnormal cardiac sounds very distinct; a mitral and aortic systolic, and a very loud, whirring aortic diastolic.

May 19th.- She continued in much the same state as last described, with intervals of ease, and with the gangrene slowly extending upwards, until to-day, when for the first time the pulse in the left upper extremity was found to be obliterated, and the limb was much colder than on the opposite side. At times there has been a good deal of delirium, but no other bad symptoms. She died quietly on the night of the 20th of May, ten weeks and six days after her admission, three weeks and six days from the symptoms of obstruction in the arteries of the lower limbs, and one day from those of obstruction of the artery of the left upper extremity. 
Autopsy (May 21st, about 14 hours post-mortem).-Body much emaciated. Over the right knee a patch of livid discoloration, with some bullæ. The right foot and leg were of a dull-red hue, apparently from gangrene. There was also a livid discoloration of the toes of the left foot and blackening of the left heel.

Lungs.-Over both lungs there were firm, old adhesions. The lungs throughout were unusually firm, but were everywhere crepitant. There were no indications of lobular pneumonia, and no plugging of the pulmonary vessels.

Heart.-Pericardium was universally adherent. The muscular tissue of the heart pale and flabby. Left side comparatively enlarged. On the auricular surface of the mitral valves, and on the lining membrane of the left auricle, were numerous soft regetations. Some of them were loosely attached, and one, about two thirds of an inch long, was attached by a narrow pedicle, and could be flapped backwards and forwards with ease.

Arteries.-The right subclavian and commencement of the right axillary, the left carotid throughout its greatest length, the common, external and internal iliac arteries, on both sides, were obstructed by firm coagula, so as to resemble firm, rounded cords. Liver normal. Fibrinous deposits in the spleen and kidneys.

Head.-Over the right hemisphere of the brain, in the cavity of the arachnoid, was a thin layer of coagulated blood, measuring about three inches from before backwards, and extending from near the longitudinal fissure downwards into the right fissure of Sylvius, where there was a considerable coagulum, as large as a filbert, adherent to branches of the middle cerebral artery, so as not to be separated when water was allowed to flow over it. Several of the more minute branches of this vessel appeared plugged ; that is, they appeared thickened, yellow, and opaque. These arteries were removed and put aside for careful examination, but were unfortunately lost. There was no softening of the cerebral substance. 
The first case offers considerable difficulty with respect to the precise date at which the plugging of the larger vessels took place. The changes observed in and around the coats of the aorta, the iliacs, and the femoral, tend to show that considerable obstruction must have been present for some time before the complete occlusion of the right and left subclavian and the posterior tibial arteries. The inflammatory process which led to the thickening of the coats at the seat of the plugging, and the formation of the large quantity of condensed fibrous tissue around, must have occupied a much longer period of time than that which intervened between the date of the symptoms of complete obstruction of the left posterior tibial artery and the fatal issue. But be this as it may, this case is of great interest, as showing the changes which obstruction, more or less complete, of the arteries produces; first, with respect to the coats of the arteries themselves, and secondly, with respect to the neighbouring structures and the parts supplied with blood by the obstructed arteries. Where there was partial obstruction, as in that portion of the aorta at the commencement of the cœliac axis, as well as at its bifurcation, the inflammation was of that kind usually denominated adhesive, and the exuded matter was susceptible of some organization. Where the plugging was more complete the inflammation was of a more intense character, and was followed by the exudation of an albumino-fibrinous material, mixed with pus, or which soon degenerated into pus. (See Plate IX.) In the second case there is less difficulty in fixing the precise date of the obstruction. The period of time which elapsed from the first appearance of the symptoms to the fatal issue was comparatively short, and there was no very evident thickening of the walls of the obstructed arteries. In both of the cases the symptoms which indicate the sudden occlusion of the arteries were wellmarked-intense pain at the seat of obstruction, and coldness and numbness, and absence of pulsation in the distal end of the affected limb. 


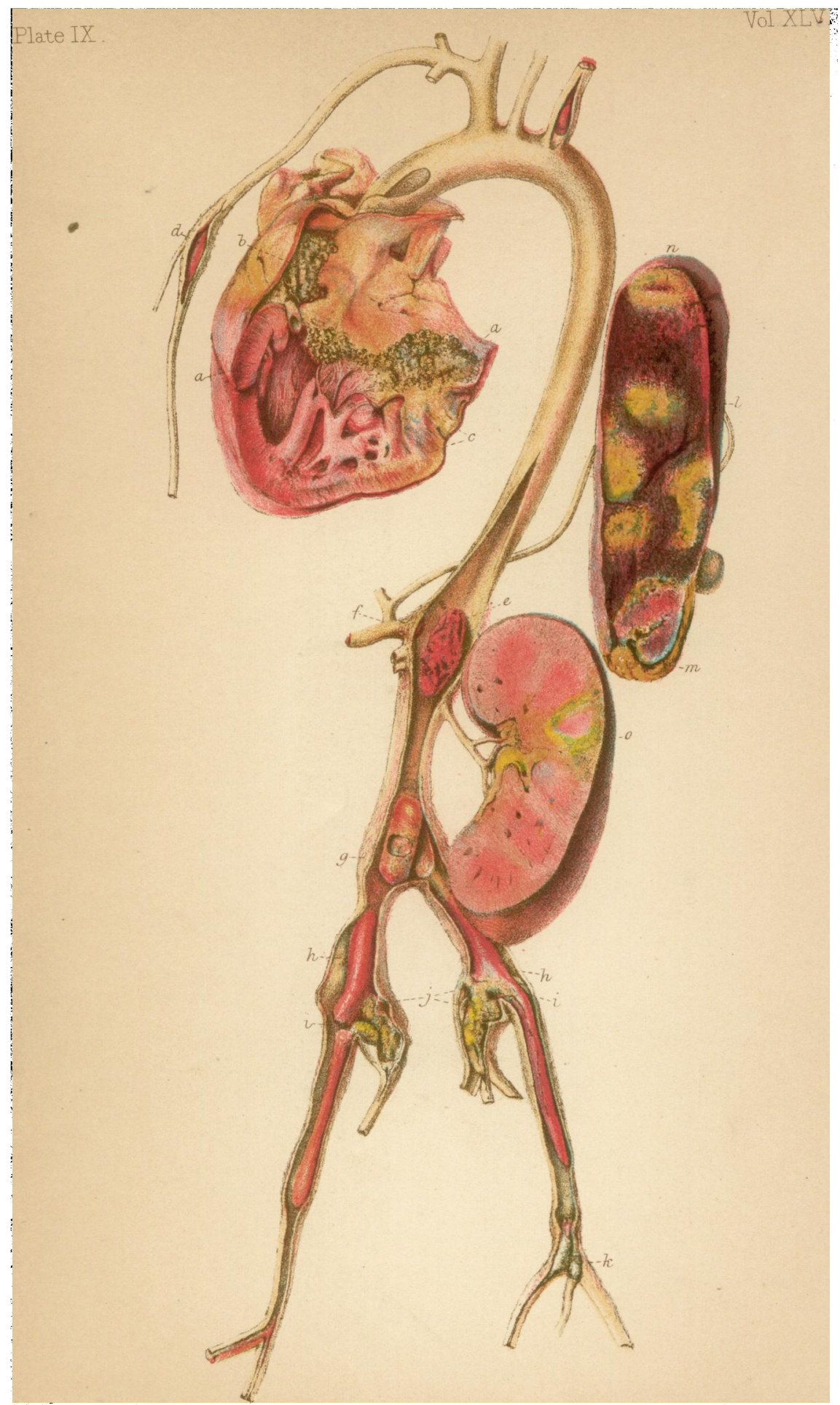




\section{DESCRIPTION OF PLATE IX.}

a. Vegetations on mitral valve.

b. Appendix filled with vegetations.

c. Wall of the ventricle infiltrated with an exudation resembling the material of which the vegetations were composed.

$d$. The coagulum in brachial artery.

e. $\quad$ in aorta, opposite to the origin of the cœliac axis.

$f$. $\quad, \quad$ in the coliac axis.

g. $\quad$ at the bifurcation of aorta.

$h$. " in the common iliacs.

$i i, j j$. $\quad$ at the bifurcation of the iliacs, and the internal iliacs.

$k$. $\quad$ in the origin of the profunda and the femoral.

In the situations marked $e, f, g, h, i, j$, and $k$, the walls of the vessels were much thickened, and a thick layer of dense, cellular tissue had formed around them. In the situations $i i, j j$, and $k$, the coats were separated by a curdy-looking, puriform matter.

The spleen, showing several so-called fibrinous deposits.

$n$. Deposit, softened and converted into a puriform mass.

n. , , , ,

o. $"$ in the left kidney. 